\title{
Formation and characterisation of ultra-sensitive surface plasmon resonance sensor based upon a nano-scale corrugated multi- layered coated D-shaped optical fibre
}

\author{
T. Allsop, R. Neal ${ }^{+}$, C. Mou, K. Kalli ${ }^{\mathrm{x}}$, S. Saied*, S. Rehman ${ }^{\#}$, D.J. Webb, P. Culverhouse ${ }^{+}$, \\ J. Sullivan", I. Bennion \\ Authors' Affiliations \\ Photonics Research Group, Aston University, Aston Triangle, Birmingham, B4 7ET, UK. \\ ${ }^{+}$University of Plymouth, Plymouth, PL4 8AA, U.K. \\ ${ }^{\mathrm{x}}$ Nanophotonics Research Laboratory, Cyprus University of Technology, Cyprus \\ *Surface Science Research Group, Aston University, Aston Triangle, Birmingham, B4 7ET, UK. \\ \# STR Fiber Technologies, Rickmansworth, Herts, UK \\ + Dept of Maths and Computing, and the Faculty of Science and Technology, \\ Plymouth Electron Microscopy Unit, University of Plymouth, Plymouth, PL4 8AA, U.K
}

\begin{abstract}
We present experimental results on the performance of a series of coated, D-shaped optical fibre sensors that display high spectral sensitivities to external refractive index. Sensitivity to the chosen index regime and coupling of the fiber core mode to the surface plasmon resonance (SPR) is enhanced by using specific materials as part of a multi-layered coating. We present strong evidence that this effect is enhanced by post ultra-violet radiation of the lamellar coating that results in the formation of a nano-scale surface relief corrugation structure, which generates an index perturbation within the fibre core that in turn enhances the coupling. We have found reasonable agreement when modelling the fibre devices. It was found that the SPR devices operate in air with high coupling efficiency in excess of 40dB with spectral sensitivities that outperform a typical long period grating, with one device yielding a wavelength spectral sensitivity of $12000 \mathrm{~nm} / \mathrm{RIU}$ in the important aqueous index regime. The devices generate surface plasmon resonances over a very large wavelength range; (visible to $2 \mu \mathrm{m}$ ) by alternating the polarisation state of the illuminating light.
\end{abstract}

\section{INTRODUCTION}

Gratings in fibres - such as long period gratings (LPGs), fibre Bragg gratings (FBGs) and tilted fibre Bragg gratings (TFBGs) - and surface plasmon resonance (SPR) based devices (in both planar and fibre configurations) may all be used to detect changes in the refractive index of a material surrounding the sensor, with potential applications in chemistry, biochemistry and biology [1-5]. The surface plasmon resonance is an important optical phenomenon that involves a resonant transfer of incident propagating light to a surfaceplasmon mode that takes the form of collective electron oscillations in a metal [6]. SPR based sensors have distinct advantages over conventional fibre sensors based upon gratings, offering especially high spectral sensitivity to changes in refractive index. These sensors can be divided into three types, based upon the kind of surface plasmon being generated at the metal/dielectric interface: the long range surface plasmon (LRSP) [3], the short range surface plasmon (SRSP) [7] and the localised surface plasmon [8]. It has been found that surface plasmon generation is very sensitive to the polarisation of the illuminating light, its wavelength, its angle of incidence on the metal surface and changes in the refractive index of the surrounding environment; it is the latter property that we exploit with our sensor design. The polarisation sensitivity can be used to detect index changes in biochemical /chemical 
reactions [9]. SPR biosensors offer the opportunity for real-time and label-free monitoring of biomolecular interactions [10]. The LRSP and SRSP obey the following dispersion relation for two homogeneous semi-infinite media:

$$
\beta=k \sqrt{\left(\frac{\varepsilon_{m} \cdot n_{s}{ }^{2}}{\varepsilon_{m}+n_{s}{ }^{2}}\right)}
$$

where $k$ is the free space wave number, $\varepsilon_{m}$ is the dielectric constant of the metal $\left(\varepsilon_{m}=\right.$ $\left.\varepsilon_{m r}+i \varepsilon_{m i}\right)$ and $n_{s}$ is the refractive index of the dielectric sample to be tested. The plasmons exist at a metal-dielectric interface and, depending on the topology of the supporting metal coating, the dispersion relation can change [8] altering their resonant wavelength condition. The situation for localised surface plasmons is more complex and there are several dependencies, such as, the size, topology or geometries of the nanostructures (for example nanoparticle dimers and nanoshells [8]).

There is a considerable body of published research relating to the use of fibre grating based devices for refractive index sensing; with the majority of the these devices, the highest index sensitivity is obtained with test sample indices approaching that of the cladding (in excess of 1.4) [11] and they generally have less sensitivity in the aqueous index regime (indices around 1.33). This problem has been addressed with the use of tilted Bragg gratings to assist in the generation of surface plasmon resonances in a fibre configuration [12].

We report on the design, fabrication and characterisation of a novel SPR fibre device that combines a lapped, D-shaped optical fibre with the deposition of multiple layers consisting of metals, semi-metals and oxide layers on fibre's lapped surface. This device is based on coupling between the core mode and localised surface plasmons (LSP) and we provide evidence that the coupling is promoted by a UV laser-induced strain field extending into the fibre core. The strain field results from the selective compaction of $\mathrm{Ge}$ in the multiple-layer structures (Table 1) containing various combinations of $\mathrm{Ge}, \mathrm{SiO}_{2}$ and silver. This selective compaction produces a surface relief structure that itself generates a grating in the form of a periodic strain field - in the core of the D-shaped fibre. This enhances the out coupling of the light to the SPR mode, which is supported by the metallic layer; a nano-scale, surface relief corrugation.

The device exhibits coupling to surface plasmons when surrounded by air and a higher index overlay material. The various multi-layered thin films deposited on the fibre provide versatility, allowing high spectral sensitivities in different external refractive index regimes and different wavelength ranges.

\section{FABRICATION AND CHARACTERISATION}

The device is constructed in three stages [12]. Firstly, a standard single mode optical fibre (SMF-28) is mechanically lapped down to within $10 \mu \mathrm{m} \pm 0.5 \mu \mathrm{m}$ from the core-cladding interface. This distance is large enough to minimise the evanescent field strength at the flat of the lapped fibre surface and to stop the coated flat of the D-shaped acting as a "mode sink" which would affect the overall dynamic range (optical power) of the sensor. Secondly, using RF sputtering, a series of coatings is deposited upon the flat of the lapped fibre; the materials used and average thicknesses are shown in Table 1. These materials and thickness were chosen after a series of calculations indicated that for a D-shaped optical fibre with a coating combining these materials, having these thicknesses, would generate surface plasmons in the infrared spectrum. We have used several combinations for the coatings of the SPR devices; these include devices with coatings of only a single semi-conductor material (germanium) which behaves as a dielectric material in the chosen wavelength range, to a complicated array of multiple germanium-silica layers, incorporating a silver overlay. The range of devices helps to provide understanding of the physical mechanism that produces the spectral 
transmission phenomena. Device 3 behaves as a more conventional SPR design, as it contains a thin layer of silver for SPR mode generation. Whereas germanium is known as a suitable material for the generation of SPR modes in the UV wavelength range [13], there is no evidence in the literature to suggest that germanium supports SPR modes in the infrared regime.

In this current study, we also note that germanium and silica layers will interact on UV laser exposure to produce germania and localised compaction [14]. We hypothesise and provide evidence that the resulting compressive field can extend to the core mode, instigating strong coupling of the guided light to the surface plasmon. This strain field causes an asymmetric radial index variation across the cross-section of the D-shaped fibre, which can be envisaged as a radially symmetric index profile in a curvilinear waveguide (by the conformal mapping technique [15]) and helps to efficiently couple light to the surface plasmons.

Table 1 Materials and their thickness used in the fabrication of the devices

\begin{tabular}{|c|c|c|c|c|c|c|}
\hline & $1^{\text {st }}$ layer & $2^{\text {nd }}$ layer & $3^{\text {rd }}$ layer & $4^{\text {th }}$ layer & $5^{\text {th }}$ layer & $6^{\text {th }}$ layer \\
\hline Materials & $\begin{array}{c}\text { Germanium } \\
\mathrm{nm}\end{array}$ & $\begin{array}{c}\text { Silica } \\
\mathrm{nm}\end{array}$ & $\begin{array}{c}\text { Silver } \\
\mathrm{nm}\end{array}$ & $\begin{array}{c}\text { Germanium } \\
\mathrm{nm}\end{array}$ & $\begin{array}{c}\text { Silica } \\
\mathrm{nm}\end{array}$ & $\begin{array}{c}\text { Silver } \\
\mathrm{nm}\end{array}$ \\
\hline Device 1 & 48 & - & - & - & - & - \\
\hline Device 2 & 48 & 48 & - & - & - & - \\
\hline Device 3 & 48 & 48 & 32 & - & - & \\
\hline Device 4 & 48 & 48 & - & 48 & 48 & 32 \\
\hline Device 5 & 24 & 48 & - & 48 & 48 & 32 \\
\hline
\end{tabular}

In the final stage, the devices are exposed to a UV-laser light diffraction pattern that is produced with a uniform phase mask having a period of $1.075 \mu \mathrm{m}$. An exposed fibre length of $3.2 \mathrm{~cm}$ was produced by scanning the UV laser (Innova ${ }^{\circledR}$ Sabre ${ }^{\circledR}$ FRED, operating at $244 \mathrm{~nm}$ ) along the fibre (scanning velocity of $0.05 \mathrm{mms}^{-1}$, and UV optical power on the fibre of $106 \mathrm{~mW}$ ) This produces a surface relief structure that has several dominant periods of approximately $1.1 \mu \mathrm{m}, 2.1 \mu \mathrm{m}$ and $4.6 \mu \mathrm{m}$. A typical example is shown in figure 1 , for device 3 for the aforementioned periods. Furthermore, figure 1 shows a typical surface prior to UV processing that has no significant spatial frequencies present when a FFT is performed. 

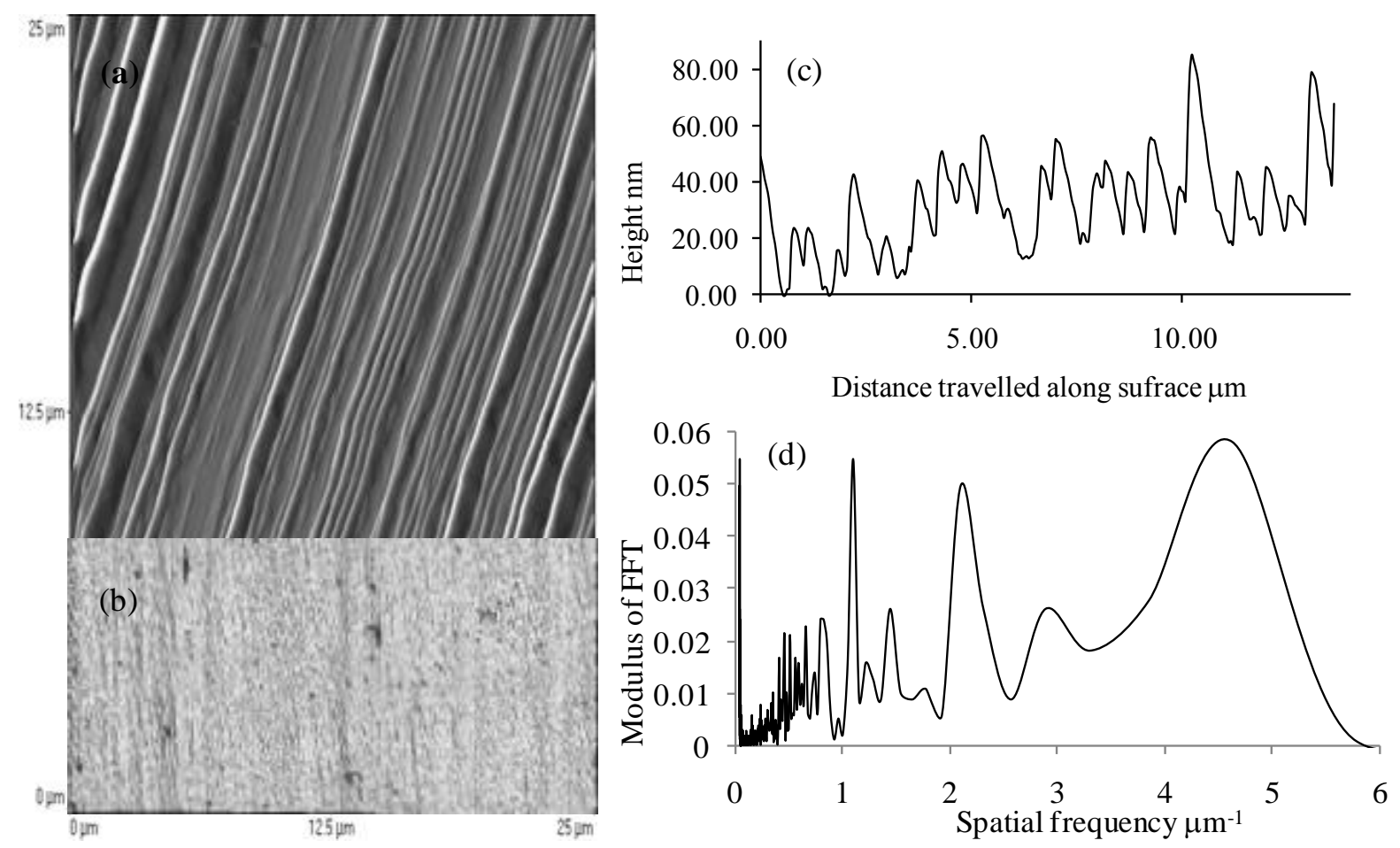

Figure 1. Images of the surface roughness of device 3 taken with an AFM (a) after UV-laser processing and (b) prior to UV-laser processing. (c) A line profile across the surface of the device post UV-laser processing. (d) A fast Fourier transform (FFT) of the line profile.

The details of the production of the surface relief modulation, which occurs in the coating during UV light exposure, is still under investigation, however it is known that UV light incident on $\mathrm{Ge} / \mathrm{GeO}_{2}$ produces photo-bleaching and compaction of the material [14]. Several publications have reported photo-induced structural changes in glasses doped with germanium or composite materials containing germanium when illuminated with UV light $[16,17]$. We envisage that spatially periodic UV illumination of the Ge and $\mathrm{SiO}_{2}$ layers in our device produces a surface corrugation due to this compaction. We suggest that the compaction creates a quasi-periodic strain field in the coating and the fibre substrate that extends into the core, which then acts to couple light from the core into the surface plasmon mode.

$\mathrm{X}$-ray photoelectron spectroscopy (XPS) is a quantitative spectroscopic technique that analyses the surface of a material, measuring the elemental composition and chemical state of the elements that exist in the surface region by determining the binding energies of the electrons. This technique was used to investigate the post fabricated multi-layered coating to look for the presence of $\mathrm{Ge} / \mathrm{GeO}_{2}$ in the coating thereby giving credence to the proposed coupling mechanism for plasmon generation that would be linked to the compositional change of the material, promoted by the UV laser exposure. A typical result for these devices is shown in figure 2. The machine used was a Thermo Fisher ESCALAB 250 Imaging XPS instrument; Figure 2 shows the germanium $2 \mathrm{p} 3$ spectrum with component due to metallic Ge and germanium oxide [18]. Table 2 shows the peak binding energy for various species along with the full width at half maximum (FWHM) at that peak binding energy, the atomic abundance (At \%) within the tested sample and the area of the peak binding energy in units of CPS.eV (eVs counts per second). Several wide scan spectra (EDX spectra) were recorded which showed the same general result, but the relative concentrations of the elements in the surface varied, indicating a certain degree of inhomogeneity. 


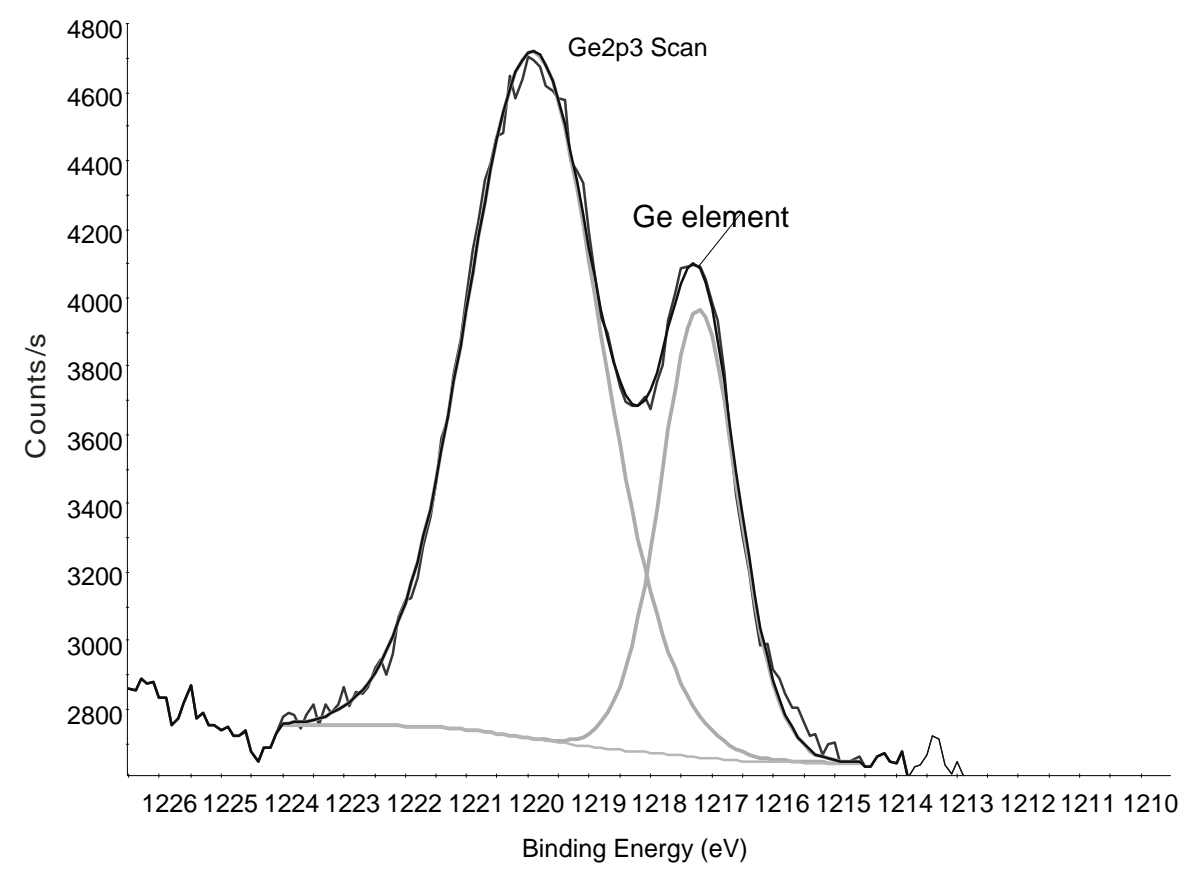

Figure 2. An example of XPS spectra of the the Ge2p3 peak from device 4, showing the presence of elemental $\mathrm{Ge}$ and $\mathrm{GeO}_{2}$, post UV-laser exposure.

Table 2 Quantitative data for the XPS

\begin{tabular}{|c|c|c|l|c|}
\cline { 2 - 5 } Name & $\begin{array}{l}\text { Peak BE } \\
\mathrm{eV}\end{array}$ & FWHM eV & $\begin{array}{l}\text { Area(P) } \\
\text { CPS. eV }\end{array}$ & At. \% \\
\hline $\mathrm{O} 1 \mathrm{~s}$ & 532.6 & 1.86 & 8573.98 & 60.2 \\
\hline $\begin{array}{c}\mathrm{Si2p} \\
\text { Oxide } \mathrm{SiO}_{2}\end{array}$ & 102.9 & 1.95 & 1139.23 & 23.1 \\
\hline $\begin{array}{c}\mathrm{Ge} 2 \mathrm{p} 3 \\
\text { Oxide }- \\
\mathrm{GeO}\end{array}$ & 1219.9 & 2.69 & 5626.39 & 10.4 \\
\hline $\begin{array}{c}\mathrm{Ge} 2 \mathrm{p} 3 \\
\text { elemental }\end{array}$ & 1217.2 & 1.54 & 2099.71 & 3.9 \\
\hline $\begin{array}{c}\text { Ag3d } \\
\text { Oxide }\end{array}$ & 368.1 & 1.01 & 1958.34 & 2.4 \\
\hline
\end{tabular}

Additional small peaks due to carbon contamination are not shown in the above table.

The optical characteristics of the fibre devices were investigated using a tuneable laser system (DWDM Passive Component Test System-IQS-12004B EXFO), which utilises a low noise sweeping tunable laser source). The system measures the transmission spectra, the optical reflection spectra and the polarisation dependent loss. As may be seen in figures 3, for the specific case of device 3, a broad spectral feature in the reflected spectrum was observed (in the spectral range from $1530 \mathrm{~nm}$ to $1600 \mathrm{~nm}$ ), suggesting that there is a step in the refractive index. This is a variation that is experienced by the core mode causing coupling to the counter-propagating core mode. This reflected feature is spectrally broad and weak; suggesting that the period of the index variation is varying along the core. A contribution to the spectral broadness may arise from strain variations brought about by spatial variation in 
the coating thickness or composition along the fibre. It was also found that the spectral features are polarisation dependent; changing the polarisation of the illuminating light changes the observed spectral features. An example of the polarisation dependence loss is shown in figure $3 \mathrm{a}$, which suggests that the index perturbation across the core is not radially symmetric. This is to be expected for a grating-like structure. It is also noteworthy that the polarisation of the light is different in figure $3 \mathrm{a}$ to $3 \mathrm{~b}$, this due to the procedure required to measure both spectra and hence the reason for any spectral differences. However, similarities can be seen in spectral location for a broad spectral feature; identified by the slope located at approximately $1590 \mathrm{~nm}$. We also observed a narrow spectral feature at close to $1565 \mathrm{~nm}$ in transmission, and in the reflection spectra in figure $3 \mathrm{~b}$. This narrow spectra feature is observed in the transmission spectra of the residual reflected light from the fibre end (Fresnel reflections) and along with the polarisation dependent loss at that spectral location. This feature is spectrally sensitive to changes in the refractive index of the surrounding medium and we believe it arises from coupling to a surface plasmon mode of the coating. Similar narrow features have been reported [19,20] involving small metallic regions sitting in a multilayered coating consisting of metal/dielectric followed by small particles of silver in a dielectric layer. In our case, the photonic/plasmonic structures show interactions between localised surface plasmons occurring on the small regions of silver that are generated on the corrugated topology created by the UV-laser processing.
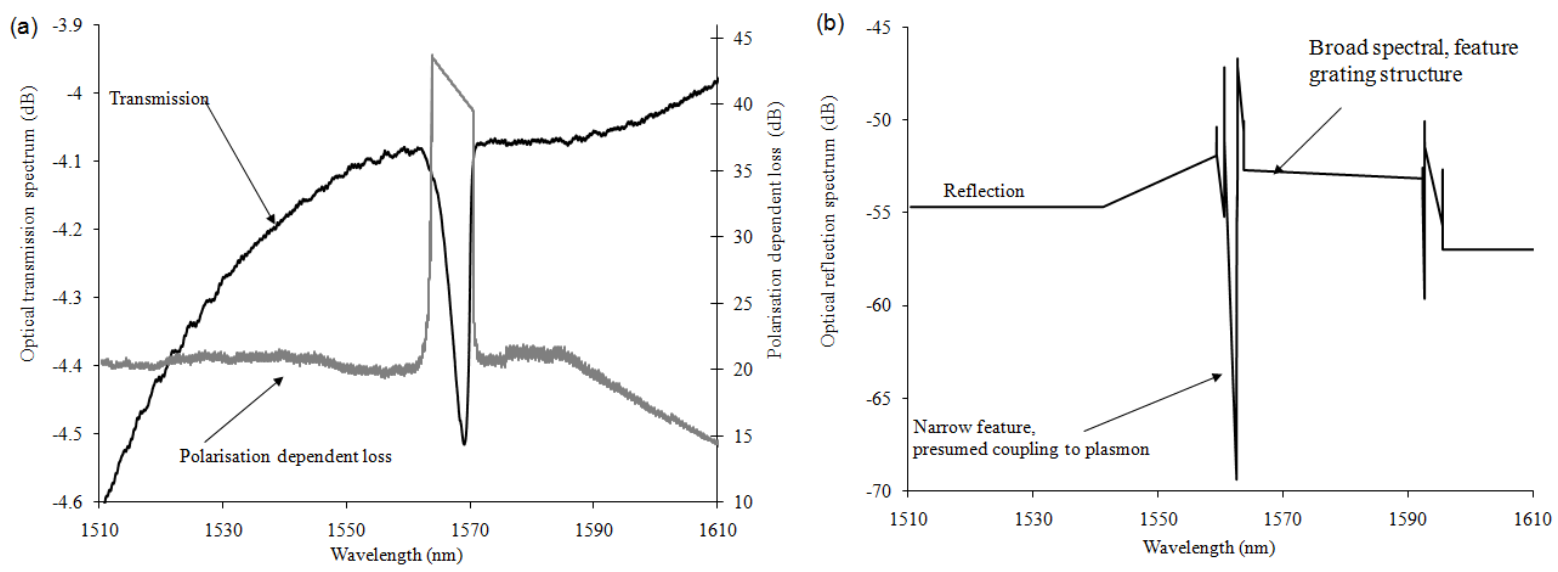

Figure 3. Examples of transmission (a) and reflection (b) spectra along with the polarisation dependent loss as a function of wavelength for fibre device 3.

The devices were further characterised by measuring the changes in their response to the polarisation properties of the illuminating light. Light from a broadband light source (several lights sources were used depending on what physical attribute - and hence what wavelength range - was being characterised) was passed through a polariser and a polarisation controller prior to illuminating the sample thereby producing light with a nominal polarisation axis. The transmission spectrum was monitored using an optical spectrum analyser (accuracy of 5pm). The change in polarisation of the illuminating light was monitored with a polarimeter (Tektronix, PAT 9000B) via a polarisation maintaining coupler, see figure 4 . This scheme was used for annealing experiments with the fibre devices placed into an insulated box with a Peltier.- 


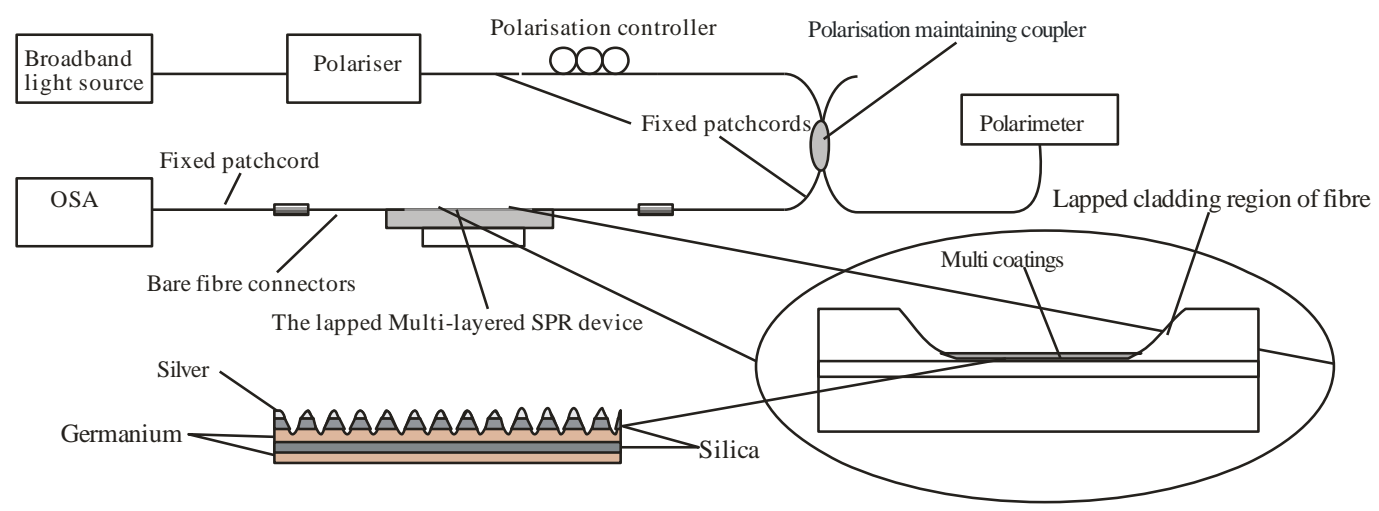

Figure 4. Scheme used for the characterisation of the devices

To ensure that a quasi-periodic strain field is generated by the coating, device 3 was annealed at $80^{\circ} \mathrm{C}$. The response of the device in transmission is shown in figure 5 for a constant temperature of $80^{\circ} \mathrm{C}$ (for a polarisation tuneable resonance at $1500 \mathrm{~nm}$, as shown in previous work [12]). Initially the spectral feature red shifted by $19.5 \pm 0.5 \mathrm{~nm}$, as the temperature increased. This spectral behaviour is followed by a slower annealing process with a blue wavelength shift of approximately $3.7 \mathrm{~nm}$ and a reduction in the optical strength of $3 \mathrm{~dB}$. It is known that the strength of the grating is given by $1-\tanh ^{2}\left(\pi \delta \mathrm{n} \gamma / \lambda_{\mathrm{B}}\right)$, where $\gamma$ represents the fraction of fibre mode power contained by the fibre's core and can be estimated using the normalised $\mathrm{V}$ parameter and $\lambda_{\mathrm{B}}=2 \mathrm{n}_{\mathrm{eff}} \Lambda$ where $\mathrm{n}_{\mathrm{eff}}$ is caused by residual stress after inscription. This low temperature annealing is characteristic of the behaviour of a standard type I UV inscribed grating and provides evidence of a grating structure in the core [21].
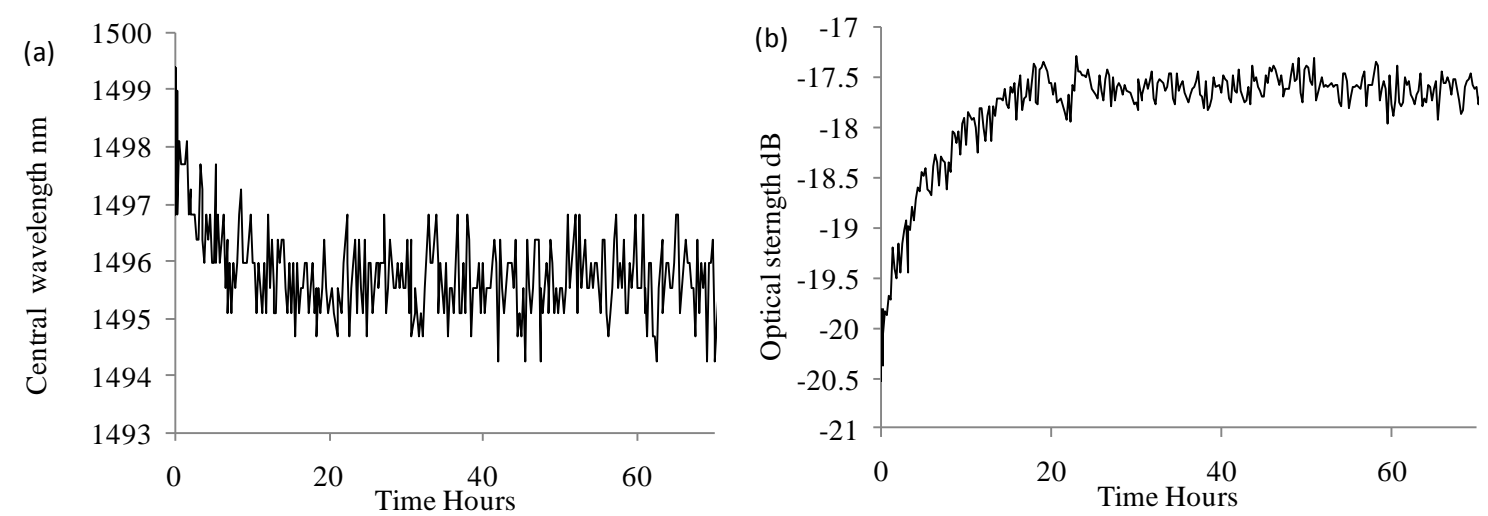

Figure 5. The spectral characteristics of a $\mathrm{Ge}_{-} \mathrm{SiO}_{2}-\mathrm{Ag}$ SPR fibre device as a function of time whilst being annealed at a temperature of $80^{\circ} \mathrm{C}$. (a) Shift in central wavelength (b) Change in optical strength of the feature measured in transmission.

An optical backscattering reflectometer (Luna OBR4400) was used to investigate fibre device 3 , which was coated with $\mathrm{Ge}_{-} \mathrm{SiO}_{2}-\mathrm{Ag}$, after UV-laser exposure. It was found that there was an index perturbation along the fibre length where the UV-laser exposure had occurred, figure $6 \mathrm{a}$ and figure $6 \mathrm{~b}$. Figure $6 \mathrm{a}$ is the shows the middle section of the total length of the fibre device, where the greatest index perturbation has occurred Figure $6 \mathrm{~b}$ shows the entire fibre device and the general back scattering of optical fibre. This piece of evidence does suggest that an index perturbation has been induced during UV-laser exposure, showing a possible coupling mechanism to the SPR and supporting the data of figure 3. 

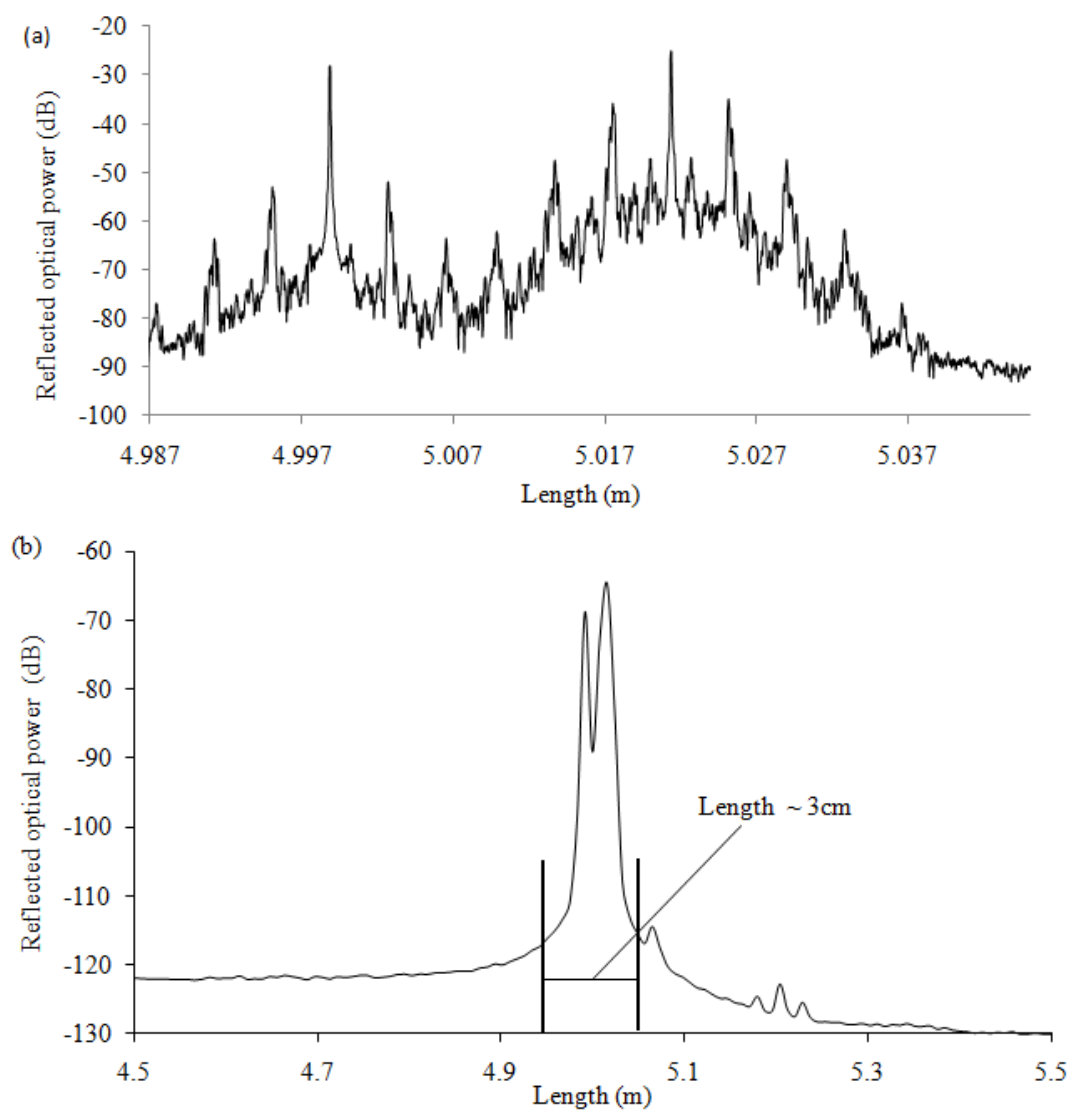

Figure 6. (a) The results obtained from using an optical backscatter reflectometer to inspect a $\mathrm{Ge}_{-} \mathrm{SiO}_{2}-\mathrm{Ag}$ device, (b) an example of the device compared to the non-perturbed fibre index

The optical properties of the devices were investigated before and after the UV-laser exposure(244nm). It was found that before UV exposure the single layer of germanium had no polarisation dependence in transmission and that there was only a small spectral variation when the surrounding medium's refractive index was varied. In particular, there were no strong spectral features within the range from $1250 \mathrm{~nm}$ to $1700 \mathrm{~nm}$ when the device was in air. Figure 7a shows the overall spectral response to the changing surrounding refractive index and figure $7 \mathrm{~b}$ shows the averaged coupling power over the wavelength range $1250 \mathrm{~nm}$ to $1685 \mathrm{~nm}$. After UV exposure strong features are observed as a function of the surrounding refractive index, see figure8. The refractive indices were provided by solutions of refractive index matching liquids (Cargille Ltd, calibrated at $589.3 \mathrm{~nm}$ and $25^{\circ} \mathrm{C}$ ). These spectral features where observed with an optical spectrum analyser (Model 86140 Agilent range from 600nm to $1700 \mathrm{~nm})$. 

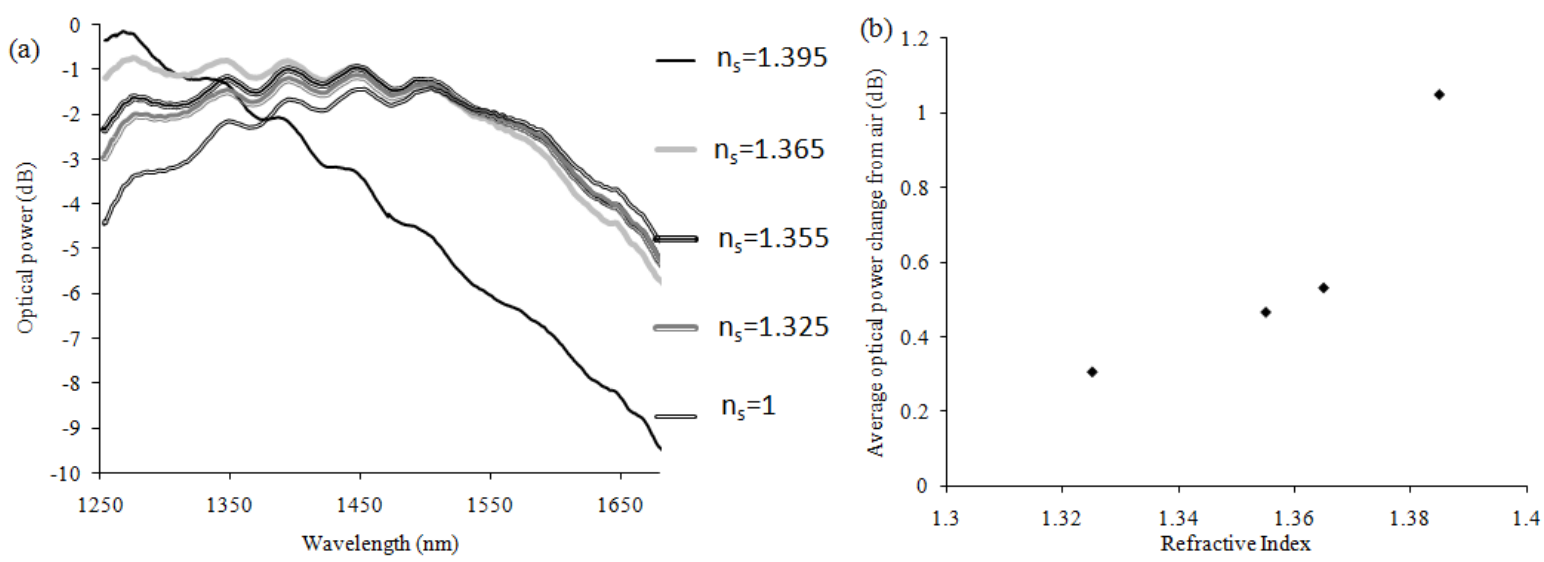

Figure 7. The observed variation in (a) the transmission spectrum of the Ge (48nm - device 1); with changes in the surrounding mediums refractive index from 1.325 to 1.395 before UV exposure (b) the optical power change (averaged over the displayed spectrum) from air as a function of refractive index before UV exposure.
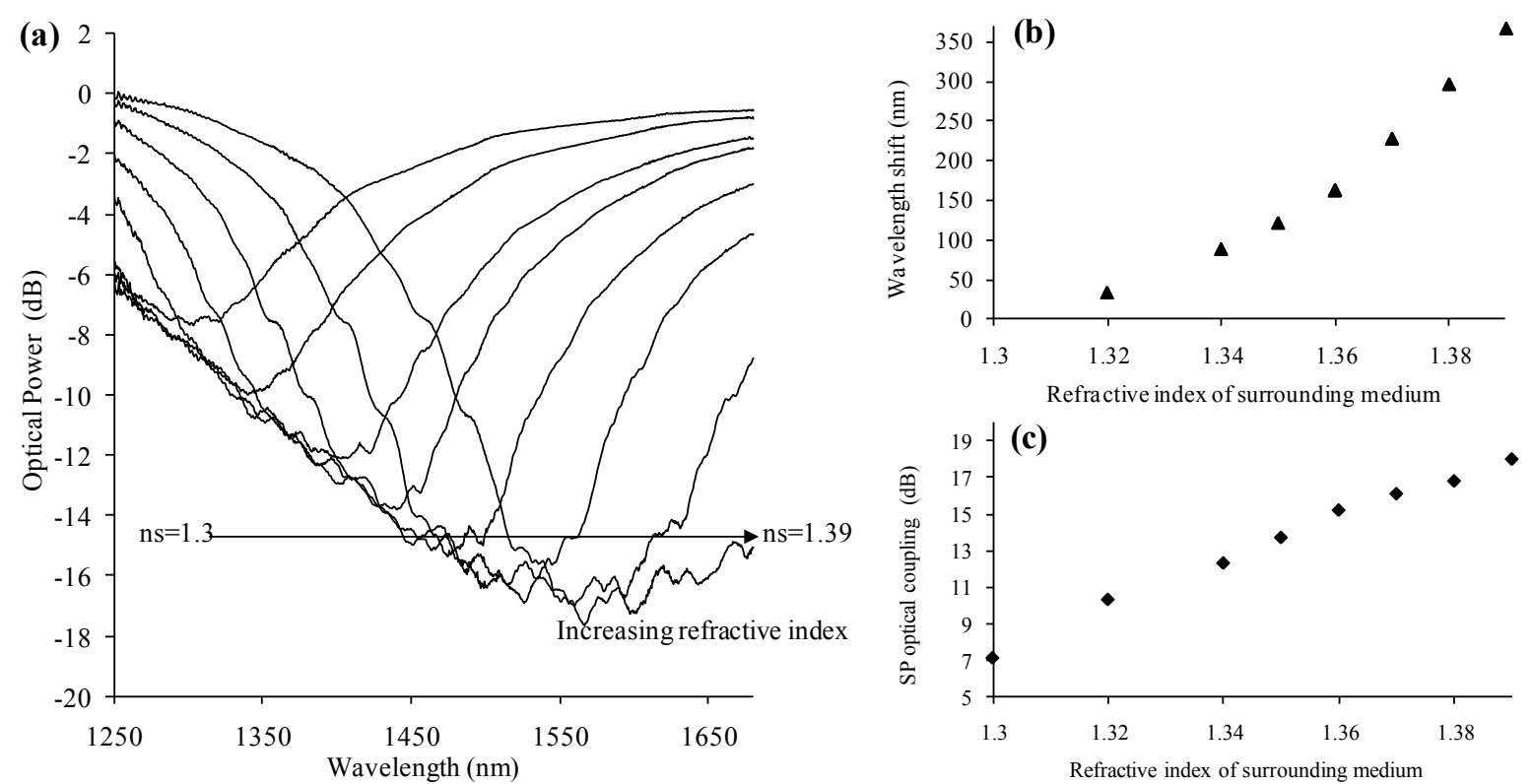

Figure 8. The observed variation in (a) the transmission spectrum of the Ge (48nm - device 1); with changes in the surrounding mediums refractive index from 1.325 to 1.39 after UVlaser exposure, (b) the wavelength shift as a function of refractive index after UV exposure, (c) the optical power change as a function of refractive index after UV exposure. 


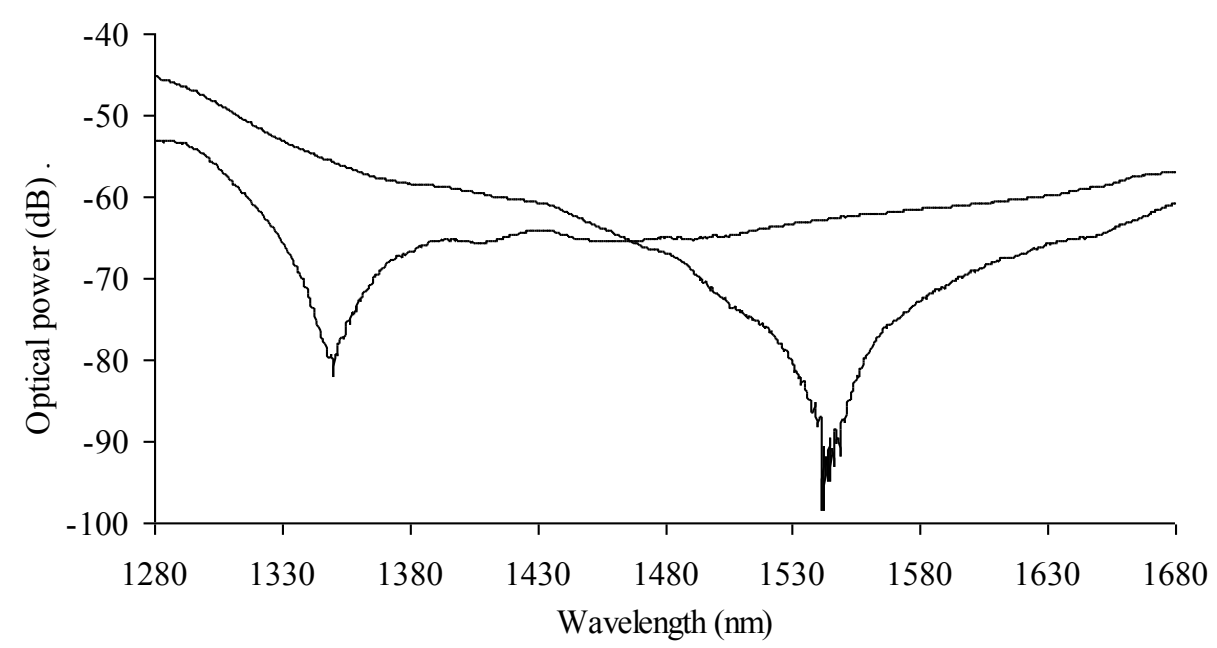

Figure 9. The transmission spectra of the device in air obtained by varying the polarisation to obtain the maximum response of the two resonances. This device consists of germanium and silica coating with thicknesses of $48 \mathrm{~nm} \mathrm{Ge}$ and $32 \mathrm{~nm} \mathrm{SiO}{ }_{2}$.

Following exposure to the UV-laser light, certain multi-layered devices (devices 2 and 3) were also investigated and showed changes in their spectral behaviour with respect to polarisation and refractive index, an example is shown in figure 9. In the case of device 1, which has no metallic coating and therefore cannot support surface plasmons, the spectral features shown were investigated using an infrared (IR) camera. We observed the outcoupling of radiation with variations in the polarisation of the guided light, figure 10 . The experimental apparatus is similar to that shown in figure 4 . The broadband light source generated a super-continuum from 400 to $2500 \mathrm{~nm}$ with an average power of $6 \mathrm{~W}$ (model SC-450-AOTF, company; Fianium Ltd). Using an acoustic optical tuneable filter, a series of channels were produced at various wavelengths with the capability of on and off switching. The light was polarised using a broadband polariser and varied with a polarisation controller and monitored with a polarimeter (Tektronix, PAT 9000B). An infrared camera system (model SU640SDWH-1.7RT, High Resolution InGaAs short wavelength infrared windowing Camera, $0.9 \mu \mathrm{m}$ to $1.7 \mu \mathrm{m}$, company; Goodrich Corporation) was situated above the fibre device to monitor any scattered light from its surface. The optical tuneable filter was tuned from $1100 \mathrm{~nm}$ to $2000 \mathrm{~nm}$ in increments of $50 \mathrm{~nm}$ and for each change in wavelength the polarisation was varied and the out-coupled scattered light was observed with the I.R. camera. Three wavelengths showed strong out-coupling: 1410nm, 1503nm and 1607nm. Figures 10a and $10 \mathrm{~b}$ show examples of the power of out-coupled light and its effective spatial location along the fibre device as a function of the change in polarisation of the illuminating light. The polarisation change is defined from the reference point of maximum coupling to the SPR mode and refers to the change in azimuth of polarisation at the polarisation controller . 

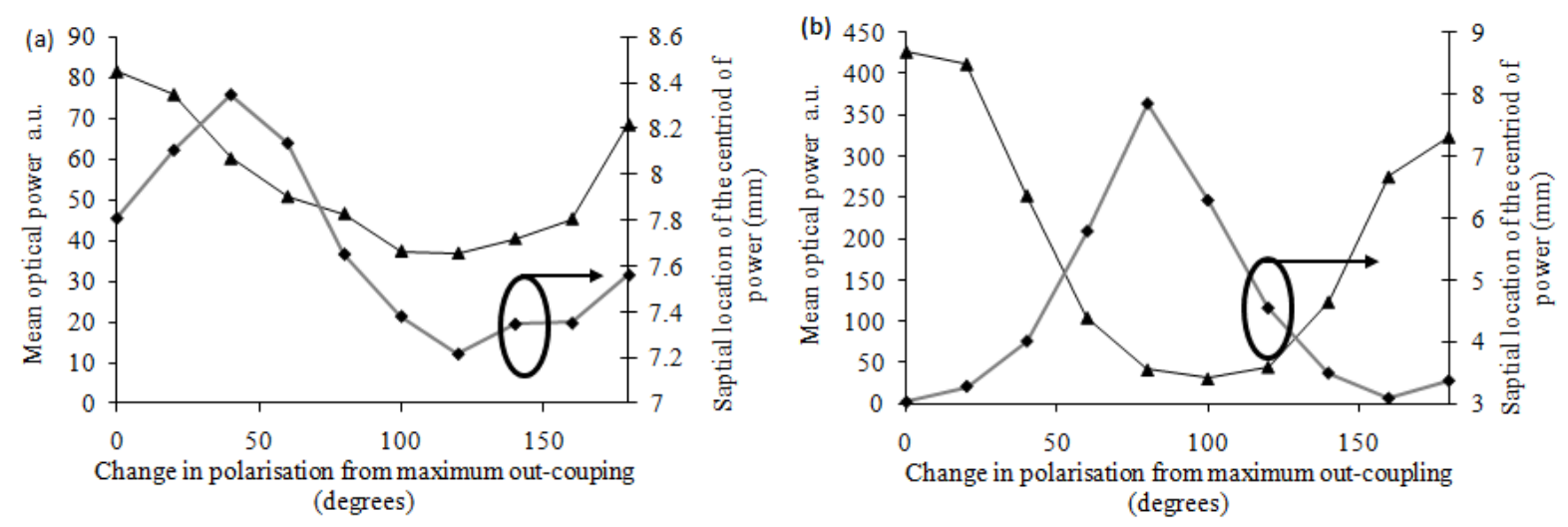

Figure 10. variation of the mean power of out-coupled light at (a) $1410 \mathrm{~nm}$ and (b) $1607 \mathrm{~nm}$ (ム) and the spatial variation (centroid location) along the flat of the D-shaped fibre ( $\downarrow$ ) as a function of the polarisation.

There have been a number of publications concerning the spectral behaviour of similar small structures (shallow lamellar metallic gratings) [22,23], which exhibit similar spectral features and a tunebility with polarisation of the incident light through a Brewster effect, with a dependence on the corrugated layer thickness, period and duty cycle [24]. This is due to the fact that the extraordinary axis along the coating surface is anisotropic and its permittivity is calculated as the arithmetic or harmonic mean value of the permittivities of the filling materials. The results in figure 10 show that at specific wavelengths for specific polarisation states the light is out-coupled, thus suggesting that what we observe is not a surface plasmon phenomenon.

It was found that all the devices had polarisation dependences and that all but the single germanium coated device demonstrated coupling to a surface plasmon, exhibiting a high extinction ratio with air as the surrounding medium. Device 4 with a Ge thickness of $48 \mathrm{~nm}$ when surrounded by air $\left(\mathrm{n}_{\mathrm{s}} \approx 1\right)$ produced two spectral transmission features at two different polarisation states of the illuminating light, with one feature appearing at a wavelength of $1300 \mathrm{~nm}$ with a maximum observed coupling of $26 \mathrm{~dB}$ and the other at a wavelength of $1560 \mathrm{~nm}$ with a maximum observed coupling of $38 \mathrm{~dB}$, see figure 11 .

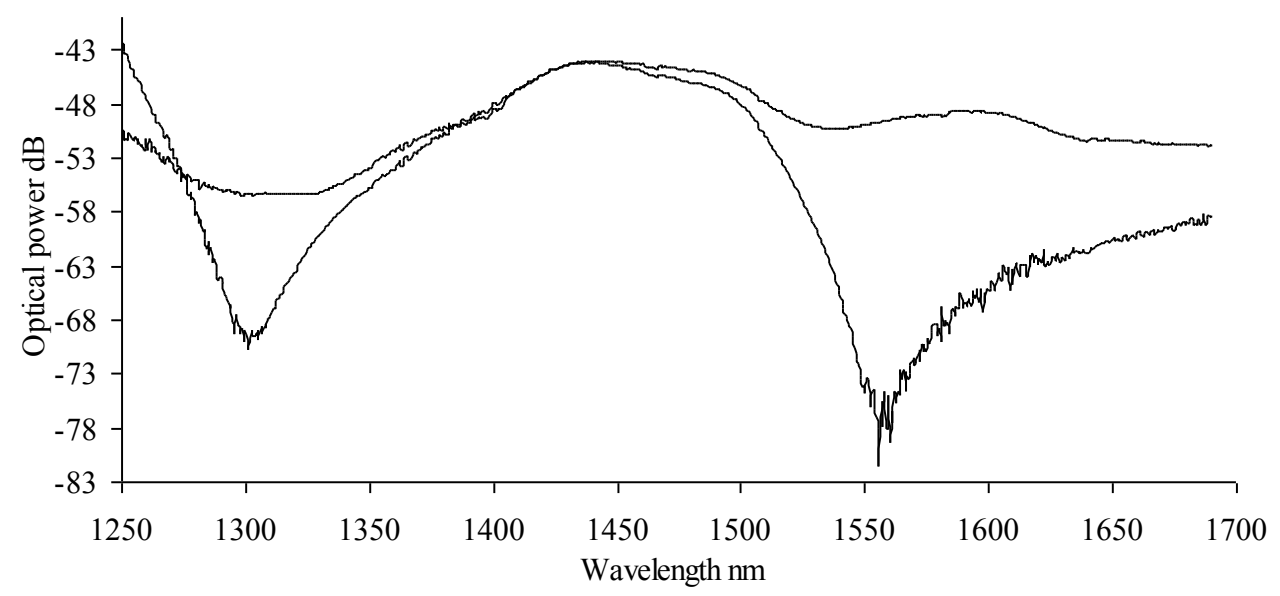

Figure 11. The transmission spectra of device $4\left(\mathrm{Ge}_{-} \mathrm{SiO}_{2}-\mathrm{Ge}-\mathrm{SiO}_{2}-\mathrm{Ag}\right)$ illuminated with two polarisation states of light with the surrounding medium being air. 


\section{REFRACTIVE INDEX SENSITIVITY}

For refractive index sensitivity measurements, the devices were placed in a $\mathrm{V}$-groove and immersed in certified refractive index (CRI) liquids (Cargille Laboratories Inc.), which have a quoted accuracy of \pm 0.0002 . The device and V-groove were carefully cleaned, washed in ethanol, and finally dried before the immersion of the device into the next CRI liquid. The Vgroove was made in an aluminium plate, machined flat to minimise bending of the fibre. The plate was placed on an optical table, which acted as a heat sink to maintain a constant temperature. The fibre device was used in conjunction with the apparatus shown in figure 4 . The spectral sensitivities of the device to the surrounding medium's index were tested prior to and after UV-laser inscription; an example is shown in figure 12. This figure shows a dramatic change in the spectral behaviour of the fibre device following UV exposure. It should also be noted that the UV processing significantly increases the strength of the coupling to the SPR from typically $3 \mathrm{~dB}$ to $25 \mathrm{~dB}$. Clearly these devices have significantly improved sensing characteristics after the UV-laser processing.
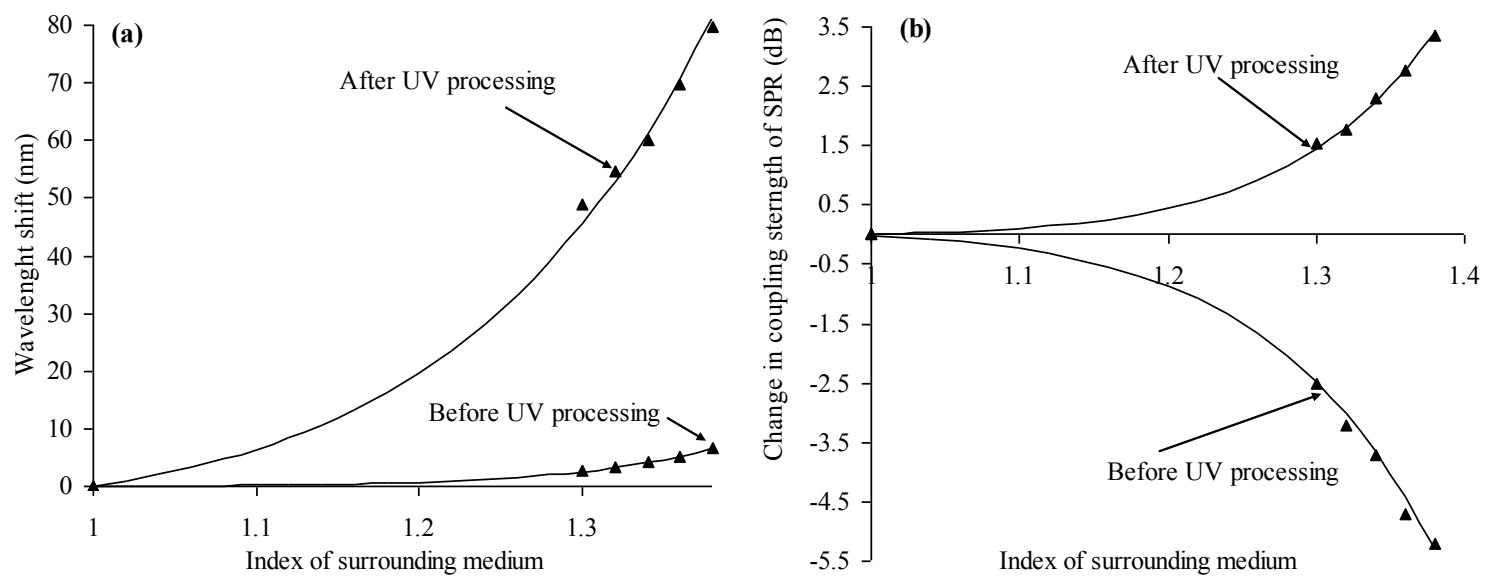

Figure 12. Example of the change in spectral sensitivities of a device with UV-laser inscription: (a) the wavelength shift, (b) the variation of coupling strength to the surface plasmon. The results shown are for device 5 .

Furthermore, varying the thickness of the first germanium layer changed the spectral performance of the device. Examples of the spectral responses of two devices with different layer thicknesses as a function of the surrounding medium's refractive index are shown in figures 13 and 14. The noise present in the transmission spectrum at the maximum coupling strength of the resonance at $1550 \mathrm{~nm}$ in figures $13 \mathrm{a}$ and $13 \mathrm{~b}$ is an artefact of the measurement system. 

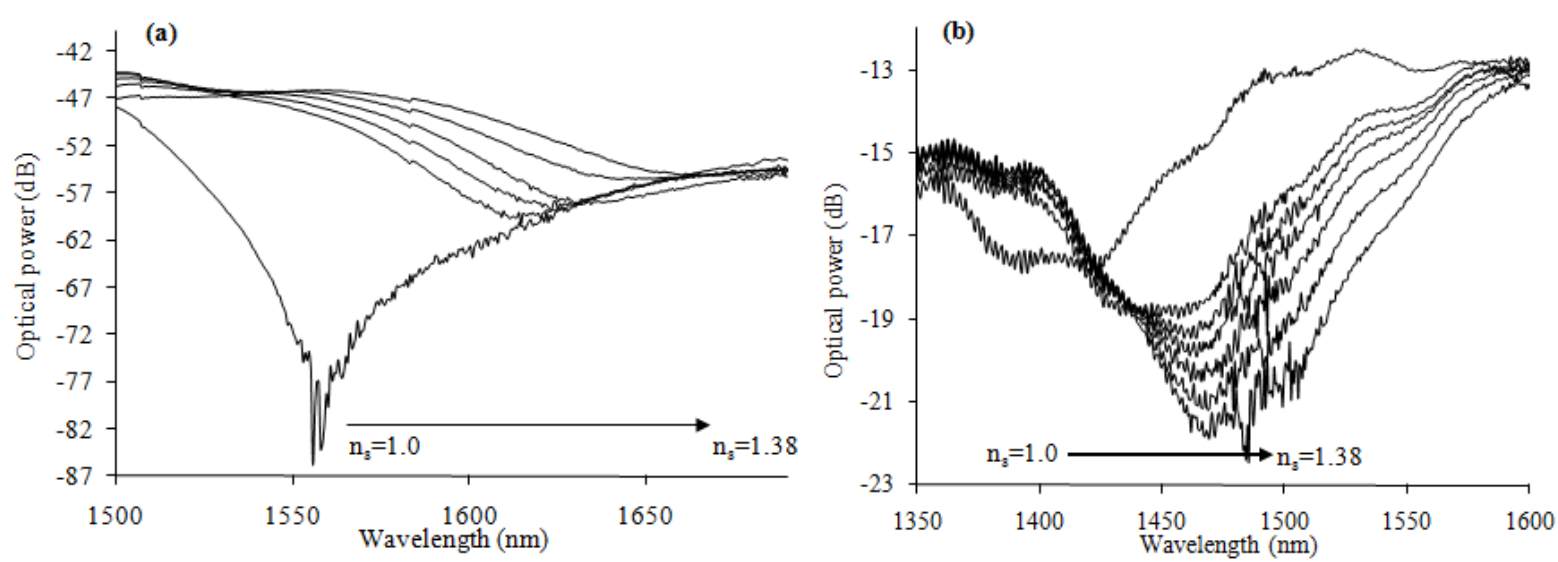

Figure 13. Transmission spectra as a function of the surrounding medium's refractive index of the $\mathrm{Ge}_{-} \mathrm{SiO}_{2}-\mathrm{Ge}_{-} \mathrm{SiO}_{2}-\mathrm{Ag}$ devices with (a) a thickness of $48 \mathrm{~nm}$ (device 4) and (b) a thickness of $24 \mathrm{~nm}$ (device 5) for the first layer of germanium.

Inspecting figures 13 and 14 shows a dramatic change in the coupling to the SPR, with the $48 \mathrm{~nm}$ device yielding a coupling strength of $43 \mathrm{~dB}$ compared to $9 \mathrm{~dB}$ for the $24 \mathrm{~nm}$ device. Such significant differences were not observed prior to UV-laser processing. The presence of greater amounts of germanium implies that more germanium oxide can be formed by UV laser exposure and therefore greater photo-induced structural changes may occur within the glass. Thus a larger quasi-periodic strain field is probably being induced in the thicker coating, which in turn increases the coupling to the surface plasmons.

It was also found that the change in thickness dramatically altered the spectral characteristics of the device after UV-laser exposure with an index sensitivity of $\Delta \lambda / \Delta n=447$ $\mathrm{nm}$ for the $24 \mathrm{~nm}$ thick device and $911 \mathrm{~nm}$ for the $48 \mathrm{~nm}$ device, figure 14 . These spectral sensitivities are comparable to other fibre based index sensors in the aqueous index regime such as LPGs [25]. This behaviour would be expected from a conventional SPR device where a specific thickness will generate optimum coupling at a particular wavelength and less efficient coupling at other spectral locations $[9,10]$.
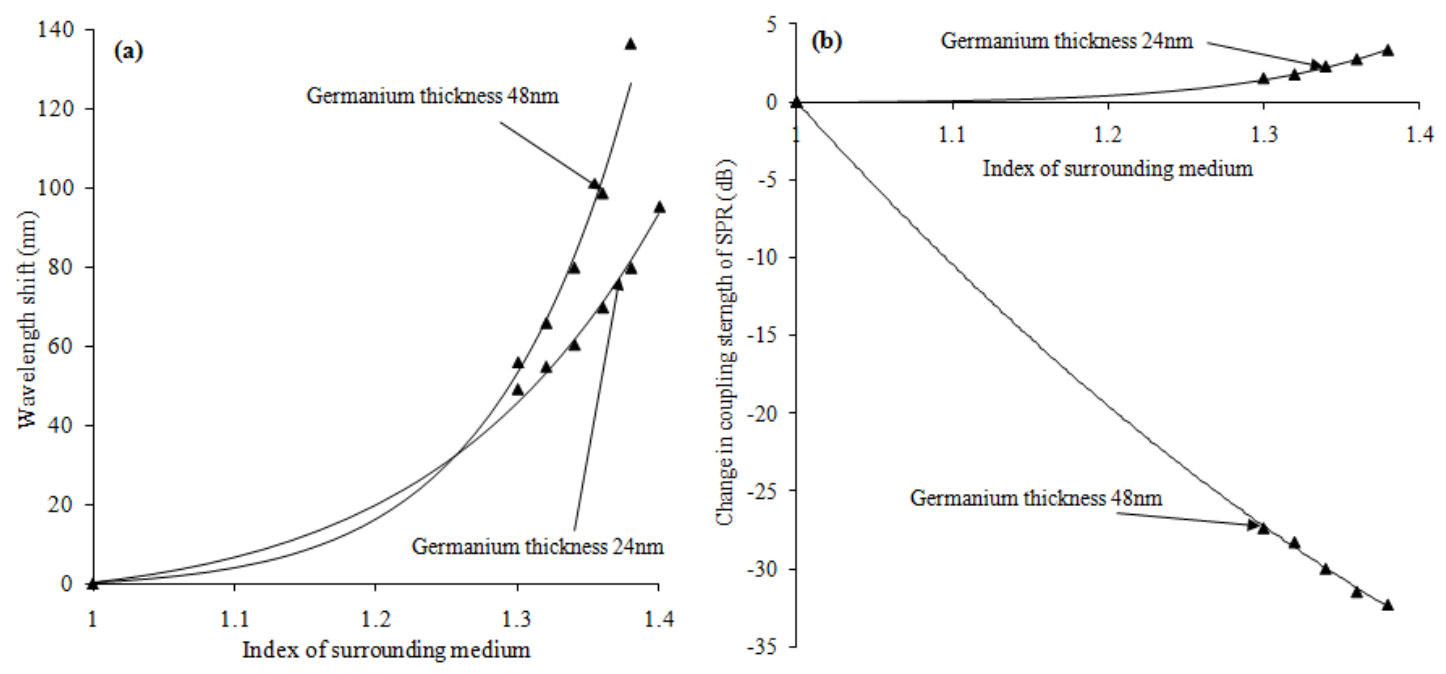

Figure 14. The spectral behaviour of devices 4 and 5 with different thicknesses of the first layer of germanium: (a) the wavelength shift, (b) the variation of coupling strength to the surface plasmon. The curves are the theoretical response discussed below. 
To obtain the theoretical results, we use the approach given in [26], which is to calculate the propagating modes of the D-shaped fibre with the coating layer, using a conformal mapping technique [27]. Each of the leaky $\mathrm{TE}_{v} / \mathrm{TM}_{v}$ modes' propagation constants yields an effective refractive index for each mode $\mathrm{n}_{\beta}$ that can be considered to have an associated scattering angle to the fibre axis $(\alpha)$, given by the ray approach; $\cos (\alpha)=\mathrm{n}_{\beta} / n_{c l}$ (where $n_{c l}$ is the refractive index of the cladding). These angles are used to give a corresponding incidence angle $(\varphi)$ of each cladding mode onto the metal/dielectric interface and thus the cladding mode wave-number projection onto that interface. Surface plasmons for the metal overlay coating (in this calculation we are assuming a smooth interface between the fibre and the coating) are generated when this wave-number projection matches the dispersion relation of the plasmons, thus [9]

$$
\frac{2 \pi}{\lambda} \sqrt{\left(\frac{\varepsilon(\lambda)_{m} \cdot n(\lambda)_{s}{ }^{2}}{\varepsilon(\lambda)_{m}+n(\lambda)_{s}{ }^{2}}\right)}=\frac{2 \pi \cdot n_{c l}}{\lambda} \sin (\varphi)
$$

Due to the post UV laser processing of the surface, the corrugated nano structures can be considered as apertures in the supporting surface plasmon material, which can be used to tune resonances in the spectral domain. For a square array of apertures the value of the momentum component of light parallel to the surface is given by [28] $k_{x}=\frac{2 \pi}{p} \cdot \sqrt{i^{2}+j^{2}}$ where $P$ is a lattice constant (the distance between the apertures), $i$ and $j$ are nonzero integer numbers representing the scattering orders from the two dimensional aperture arrays. The resonant condition for the localised surface plasmons that needs to be satisfied for a lattice structure is given by [29] and approximated with

$$
\lambda_{\text {res }}=\frac{p}{\sqrt{i^{2}+j^{2}}} \sqrt{\left(\frac{\varepsilon(\lambda)_{m} \cdot n(\lambda)_{s}^{2}}{\varepsilon(\lambda)_{m}+n(\lambda)_{s}^{2}}\right)}
$$

where $\varepsilon_{m}$ is the dielectric constant of the metal $\left(\varepsilon_{m}=\varepsilon_{m r}+i \varepsilon_{m i}\right)$ and $n_{s}$ is the refractive index of the surrounding medium. The value for the surrounding medium is estimated using the Maxwell-Garnett theory [30]. To obtain the resonant condition for the plasmons, the surface corrugation is envisaged as a one dimensional array of parallel slits. Thus, equation (3) is used with only one of the integer numbers $(i)$ to represent the scattering order of the surface corrugation structure. This yields an effective dielectric function as a function of the fractional volume of the metal/semi-conductor within an effective layer. The theoretical spectral transmission response of the device is obtained by calculating the reflected intensity from the metallic interface at various wavelengths. The quantitative description of the minimum of the reflected intensity $R$ for a SPR can be obtained from Fresnel's equations for a multilayered system; the reflectivity $R$ for $p$-polarised light is given by [9]. In this work the effective change of the surrounding index is determined by a sensing volume, which is based upon the area of the coating multiplied by the spatial extension of the evanescent field associated with the surface plasmon perpendicular to the coating's surface and the fractional change of the index in that volume; a more detailed description is given in [31] and [26]. In the case of coatings that only have a dielectric/semi-conductor (germanium) overlay, the above approach is used to obtain the propagation constant of the modes. A response is calculated using Fresnel's equations with an estimate of an effective index of the textured post UV processed germanium by the Maxwell-Garnett theory.

Figure 15a shows an example of the phase matching of some of the localised surface

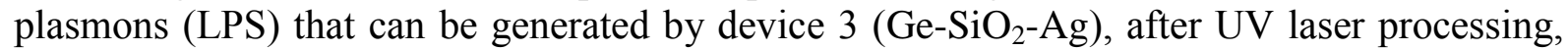
as a function of refractive index. for the surface topology shown in figure 1 and the associated 
index spectral sensitivities $(\Delta \lambda / \Delta \mathrm{n})$ of two of LPS is shown in figure $15 \mathrm{~b}$. The estimation of the phase matching of the LPS is achieved by calculating the FFT from the surface topology of the device from the AFM data, then using the spatial resonances as the spatial lattices for the calculations to spectral predict the LPS [29]. It is assumed that we have coupling between the $\mathrm{HE}_{1,1}$ and higher order modes to several localised surface plasmons with scattering integer order $(i=1)$ and for the dominant periods of approximately $2.1 \mu \mathrm{m}$ and $4.6 \mu \mathrm{m}$ found in the FFT. The spectral wavelength and coupling strength sensitivities have been investigated in the low refractive index regime $(\sim 1.01$ to $\sim 1.1)$. Whilst there are differences between the experimental data and the modelling [32,33] this may be due to the dispersion relationship assumed for the D-shaped fibre in the modelling. The dispersion relationship is sensitive to the precise fibre geometry [27].
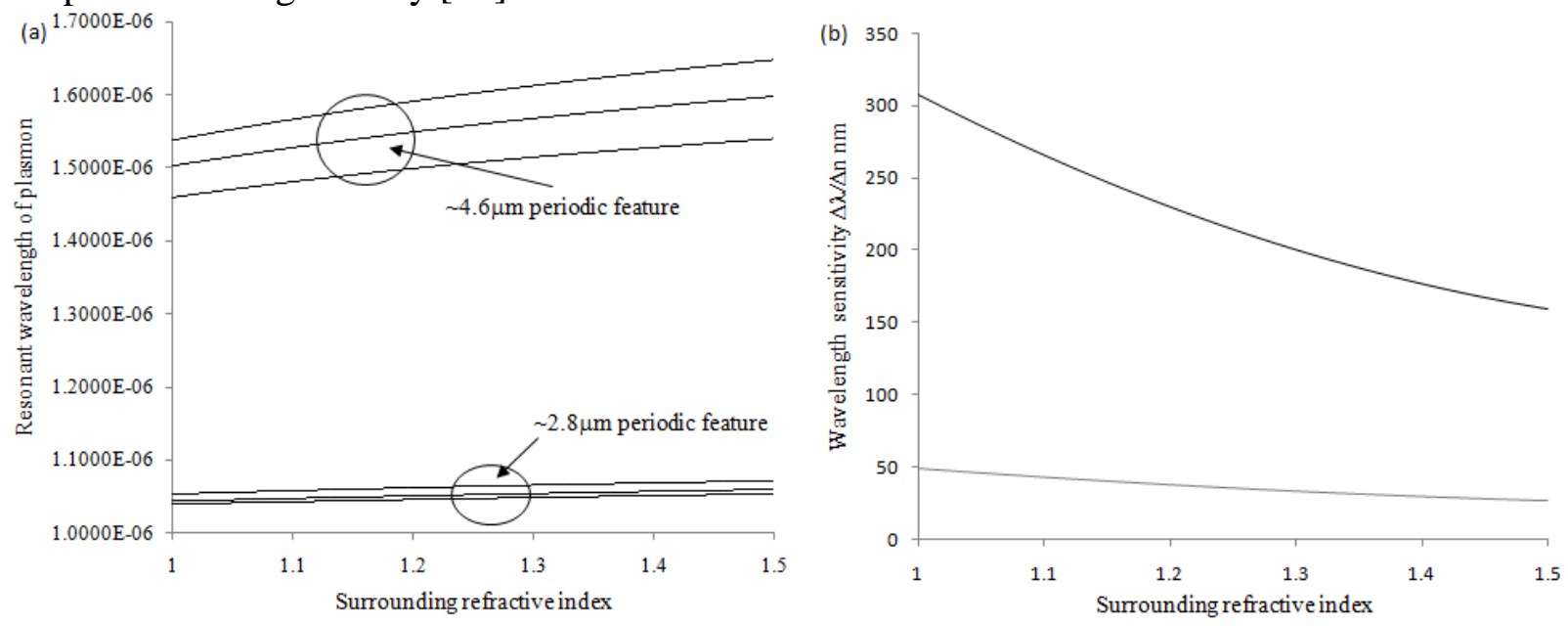

Figure 15. (a) The theoretically predicted wavelength shift of some of the surface plasmon resonances of a $\mathrm{Ge}_{-} \mathrm{SiO}_{2}-\mathrm{Ag}$ coated device (device 3 ) with the spatial lattices given by the FFT of figure 1a, and with the scattering integer order $(i=1)$. The top line is coupling with the $\mathrm{HE}_{1,1}$ mode, followed by coupling to the $\mathrm{HE}_{1,4}$ and $\mathrm{HE}_{1,8}$ modes. (b) The predicted spectral wavelength sensitivities of device 3 , assuming the coupling to the SPR is from the $\mathrm{HE}_{1,1}$ mode.

A summary of the index sensing results is shown in figures 16 and 17. The spectral sensitivities for the various coated SPR devices are compared to a typical LPG in a single mode fibre, for which the evanescent electric fields of the cladding modes interact with the surrounding medium $[1,20]$. The summary of sensitivities is based upon a combination of theoretical results (for indices below 1.3) and experimental data as a means to show the potential of these devices, and the dramatic improvement in sensitivity over LPGs. These figures show that the LPG is significantly less sensitive than the Ge coated, $\mathrm{Ge}_{-} \mathrm{SiO}_{2}-\mathrm{Ag}$ coated and $\mathrm{Ge}_{-} \mathrm{SiO}_{2}-\mathrm{Ge}_{-} \mathrm{SiO}_{2}-\mathrm{Ag}$ coated devices in the aqueous index regime, and also is outperformed by the $\mathrm{Ge}-\mathrm{SiO}_{2}$ coated, $\mathrm{Ge}-\mathrm{SiO}_{2}-\mathrm{Ag}$ coated and $\mathrm{Ge}_{-} \mathrm{SiO}_{2}-\mathrm{Ge}_{-} \mathrm{SiO}_{2}-\mathrm{Ag}$ coated devices in the low index regime. Thus our devices yield potentially higher index resolution than LPGs in both index regimes, showing their versatility and potential for high spectral sensitivity over a wide range of indices. 


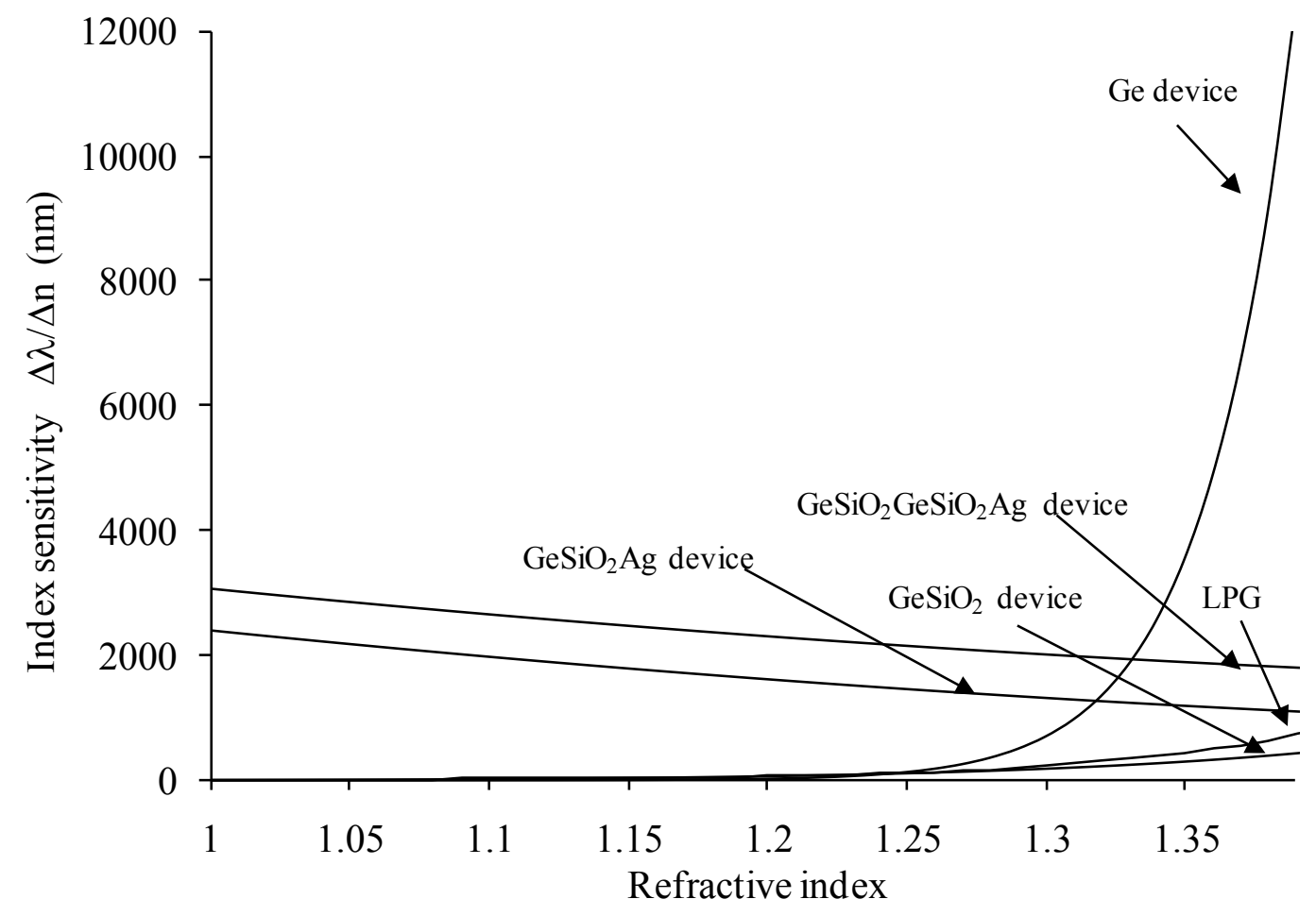

Figure 16. Wavelength spectral sensitivity comparison of the four different types of coated fibre devices as a function of refractive index, along with a long period grating (period = $240 \mu \mathrm{m}$, length $5 \mathrm{~cm}$ ). The figure combines both experimental results with certified refractive index liquids from 1.3 to 1.4 and theoretical results for refractive indices from 1.0 to 1.3.

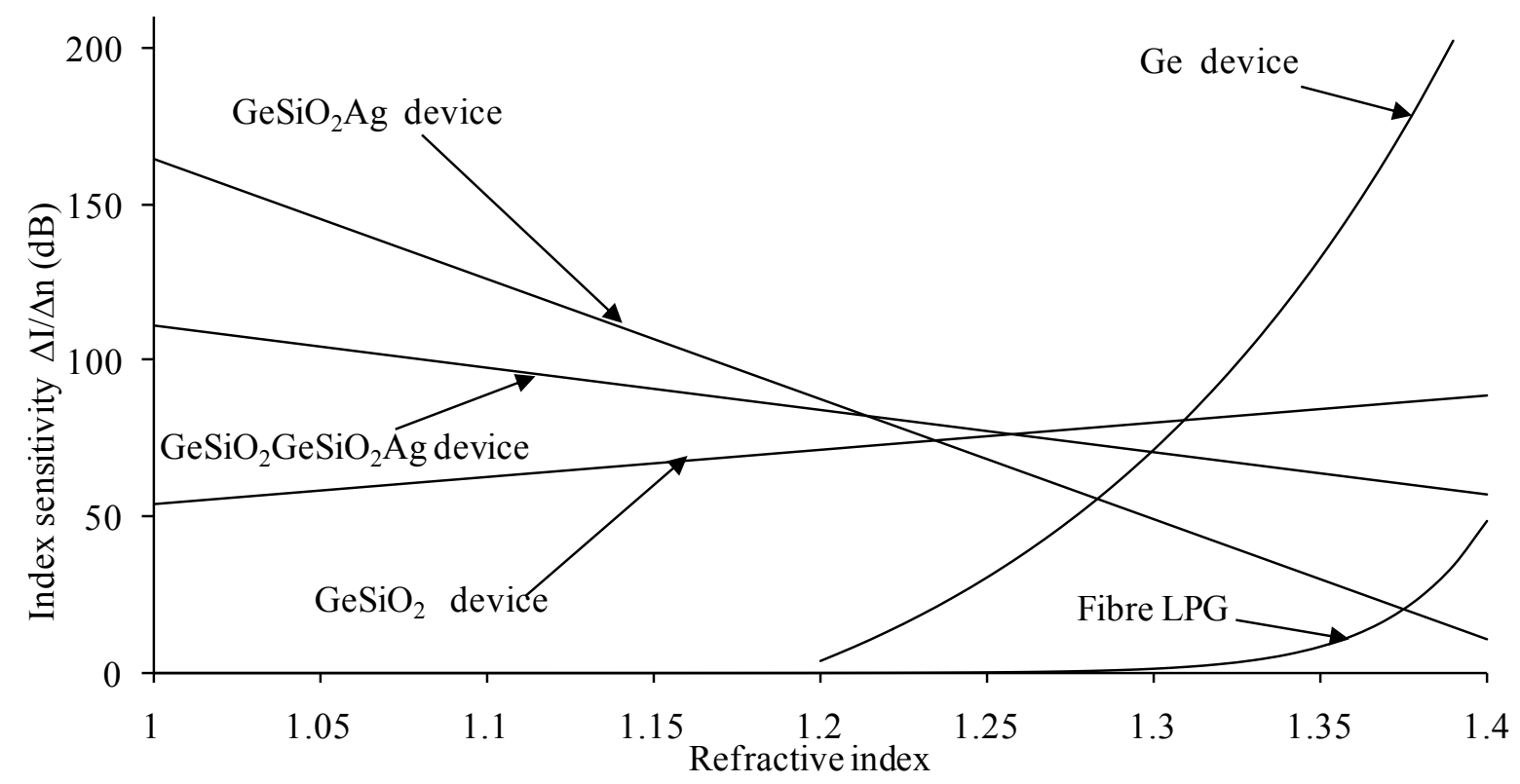

Figure 17. Optical power sensitivity comparison of the four different types of coated fibre devices as a function of refractive index along with a long period grating (period $=240 \mu \mathrm{m}$, length $5 \mathrm{~cm}$ ). The figure combines both experimental results with certified refractive index liquids from 1.3 to 1.4 and theoretical results for refractive indices from 1.0 to 1.3. 


\section{Conclusion}

This research presents the results governing a set of novel surface plasmon devices based on a combination of multilayered thin films deposited on the flat side of a lapped D-shaped optical fibre, and a UV-laser induced nano-scale, surface relief grating. The coupling mechanism between the core mode and localised surface plasmons is promoted by the surface relief structure. Evidence has been presented indicating that a strain field is generated through photo-structural changes in the multi-layered coating, on UV-laser exposure. The coupling is characterized by a broad spectral feature in the optical spectrum (figure3). X-ray photoelectron spectroscopy has shown the presence of $\mathrm{GeO} 2$ in the coatings, which is known to result in photo-structural changes on exposure to UV light, producing an index perturbation in the location of the UV processing, whilst significantly increasing coupling to the SPR modes. Thicker germanium layers were shown to generate larger SPR coupling. The majority of the SPR fibre devices operate in air, with high coupling efficiencies in excess of 40dB. The devices yielded different index sensitivities, with the single Ge coated device possessing the highest sensitivity in the aqueous regime, whereas theoretical predictions suggest that the $\mathrm{Ge}$ $\mathrm{SiO} 2-\mathrm{Ag}$ and $\mathrm{Ge}-\mathrm{SiO} 2-\mathrm{Ge}-\mathrm{SiO} 2-\mathrm{Ag}$ coated devices should be the most sensitive at low indices. All the devices outperform LPGs in these regimes with the exception of $\mathrm{Ge}-\mathrm{SiO} 2$ in the aqueous regime. In general, these types of SPR fibre devices are versatile and appear to have potential applications for both aqueous and gas (low refractive index) sensing.

\section{References}

[1] S. Vasilev et al, "Long-period refractive index fibre gratings: properties, applications and fabrication techniques", Proc. SPIE 4083, pp.212-223, 2000.

[2] K. Schroeder et al, “A fibre Bragg grating refractometer", Meas. Sci. Technol. 12, pp.757764, 2001.

[3] M. Piliarik et al, "Surface plasmon resonance sensor based on a single-mode polarisationmaintaining optical fiber", Sensors and Actuators B, Vol. 90, pp.236-242, 2004

[4] J. Homola, "Surface plasmon resonance sensors: review", Sensors and Actuators B, Vol. 54 , pp.3-15, 1999.

[5] C. Holmes, et al, "Excitation of surface plasmons using tilted planar-waveguide Bragg gratings", IEEE Jn. Photonics. Vol.3(5) pp.777-788, 2011.

[6] S. Patskovsky et al, "Properties and sensing characteristics of surface plasmon resonance in infrared light", J. Opt. Soc. Am. A, Vol.20, No. 8, pp.1644-1650, 2003.

[7] R. Slavík J. Homola, "Ultrahigh resolution long range surface plasmon-based sensor", Sensors and Actuators B, Vol.123, No. 1, pp.10-12, 2007.

[8] A. J. Haes and R. P. Van Duyne, "A unified view of propagating and localized surface plasmon resonance biosensors", Anal. Bioanal. Chem. Vol. 379, pp.920, 2004

[9] "Surface Plasmons on smooth and Rough Surfaces and on Gratings", H. Raether,, eds. (Academic, New York, 1997),

[10] J. M. Brockman et al, "Surface Plasmon Resonance Imaging Measurement of Ultra-thin Organic Films", Annu. Rev. Phys. Chem., Vol. 51, pp.41-63, 2000.

[11] M. Iga et al, "Gold thickness dependence of SPR-based hetero-core structured optical fiber sensor", Sensors and Actuators B, Vol. 106, pp.363-368, 2005. 
[12] T. Allsop et al, "Surface Plasmon Resonance Generation Utilising Gratings for Biochemical Sensing”, OFS-18 Cancun Mexico, Biological and Medical Sensors, paper WA4, Oct 2006.

[13] J. J. Cowan, "Surface plasmon resonance radiation from Germanium", Thin Solid Films, Vol. 28, pp.125-130, 1975

[14] D.L. Williams et al " UV spectroscopy of optical fibres and preforms “, Proc. Soc. Photo. Opt. Instrum. Eng., Vol. 1516, pp29, 1991.

[15] M. Heiblum and J. H. Harris, "Analysis of curved optical waveguides by conformal transformation,” Quantum Electron. QE-11_Vol.2_, pp.75-83, 1975_.

[16] D. Gonbeau, V. Pamukchieva, R. Dedryvere, E. Skordeva, D. Arsova, "Photoinduced changes in the valence band states $\mathrm{Ge}_{\mathrm{X}} \mathrm{As}_{40-\mathrm{X}} \mathrm{S}_{60}$ of thin films", Jn of Optoelectronics and Advanced Materials Vol. 7, No. 1, pp. 341 - 344, 2005

[17] H Tsuji1, N Arai, N Gotoh, T Minotani, K Kojima, K Adachi, H Kotaki, T Ishibashi, Y Gotoh and J Ishikawa, "Germanium nanoparticles formed in silicon dioxide layer by multienergy implantation and oxidation state of Ge atoms", Jn of Physics: Conference Series (Int. Conf. Nanoscience and Technology (ICN\&T 2006)) Vol. 61, pp.1196-1201, 2007

[18] Jolly, William L., Modern Inorganic Chemistry (2nd Edition ed.). New York: McGrawHill., 1991

[19] T. Kume, et al, "Interaction between localized and propagating surface plasmons: Ag fine particles on Al surface”, Solid State Comms., Vol. 93, No. 2, pp.171-175, 1995

[20] T. Kume, et al, "Light emission from surface plasmon polaritons mediated by metallic fine particles", Phys. Rev. B, Vol. 55, No. 7, pp.4774-4782, 1997

[21] T. Erdogan, V. Mizrahi, P. J. Lemaire, D. Monroe, "Decay of ultraviolet-induced fiber Bragg gratings", J. Appl. Phys., Vol. 76, No. 1, pp.73-80, 1994.

[22] E. Popov, S. Enoch, N. Bonod, "Absorption of light by extremely shallow metallic gratings: metamaterial behaviour, "Optics Express, Vol. 17, No. 8, pp.6770, 2009.

[23] N. Bonod and E. Popov, "Total light absorption in a wide range of incidence by nanostructured metals without plasmons," Opt. Lett. Vol. 33, pp.2398-2400, 2008.

[24] Z. Wang,_ T. Sang, L Wang, J. Zhu, Y. Wu, L. Chen, "Guided-mode resonance Brewster filters with multiple channels", Appl. Phys. Letts. Vol.88, 251115_2006_

[25] T. Allsop and L. Zhang and I. Bennion, "Detection of organic aromatic compounds in paraffin by a long period fiber grating optical sensor with optimised sensitivity", Opt. Comm. Vol. 191 no. 3-6, pp. 181-190, 2001.

[26] T. Allsop et al, "The exploitation of multilayer coatings for infra-red surface plasmon resonance fibre sensors", Appl. Optics., Vol.48, pp. 276-286, 2009

[27] "Optical Fibre Waveguide Analysis", C. Tsao eds. , (New York: Oxford, 1992).

[28] T. W. Ebbesen, H. J. Lezec, H. F. Ghaemi, T. Thio, and P. A. Wolff, "Extraordinary optical transmission through sub-wavelength hole arrays", Nature, Vol. 391, pp667, 1998.

[29] A. G. Brolo, R. Gordon, B. Leathem, and K. L. Kavanagh, "Surface plasmon sensor based on the enhanced light transmission through arrays of nanoholes in gold films," Langmuir Vol. 20, pp.4813, 2004

[30] D. E. Aspnes, J. B. Theeten, F. Hottier, " Investigation of effective-medium models of microscopic surface roughness by spectroscopic ellipsometry," Phys. Rev. B 20, No. 8, pp.3292 - 3302, 1979.

[31] T. Allsop, R. Neal, S. Rehman, D.J. Webb, D. Mapps, I. Bennion, “The chacterisation of infra-red Surface Plasmon Resonances generated from fibre optical sensor utilising tilted Bragg gratings", JOSB Vol. 25, No. 4, pp.481-490, 2008.

[32] T. Allsop R. Neal, S. Rehman, D.J. Webb, D. Mapps, I. Bennion, "Gas Sensing using a Surface Plasmon Resonance Fibre Device”, OFS-20, Post-deadline session, Edinburgh, United Kingdom, 2009 
[33] T. Allsop, R. Neal, E. M. Davies, C.Mou, P. Brown, S. Rehman, K. Kalli, D.J. Webb, P. Calverhouse, I. Bennion, "Low refractive index gas sensing using Surface Plasmon Resonance fibre device", Measurement and Science Technology, Vol. 21, No. 9, pp.4029, 2010 